\title{
Effect of gravitational radiation reaction on nonequatorial orbits around a Kerr black hole
}

\author{
Fintan D. Ryan \\ Theoretical Astrophysics, California Institute of Technology, Pasadena, California 91125
}

(Received 25 October 1995)

\begin{abstract}
The effect of gravitational radiation reaction on orbits around a spinning black hole is analyzed. Such orbits possess three constants of motion: $\iota, e$, and $a$, which correspond, in the Newtonian limit of the orbit being an ellipse, to the inclination angle of the orbital plane to the hole's equatorial plane, the eccentricity, and the semimajor axis length, respectively. First, it is argued that circular orbits $(e=0)$ remain circular under gravitational radiation reaction. Second, for elliptical orbits (removing the restriction of $e=0$ ), the evolution of $\iota, e$, and $a$ is computed to leading order in $S$ (the magnitude of the spin angular momentum of the hole) and in $M / a$, where $M$ is the mass of the black hole. As $a$ decreases, $\iota$ increases and $e$ decreases.
\end{abstract}

PACS number(s): 04.25.Nx, 04.30.Db

\section{INTRODUCTION}

The Earth-based Laser Interferometer Gravitational Wave Observatory- (LIGO-)VIRGO [1,2] network of gravitational wave detectors (which is now under construction) and the European Space Agency's planned space-based Laser Interferometer Space Antenna (LISA) [3] will be used to search for and study the gravitational waves from "particles," such as neutron stars and small black holes, spiraling into massive black holes (mass $M$ up to $\sim 300 M_{\odot}$ for LIGO-VIRGO and up to $\sim 10^{7} M_{\odot}$ for LISA). To search for the inspiral waves and extract the information they carry will require templates based on theoretical calculations of the emitted waveforms, which in turn require a detailed understanding of how radiation reaction influences the orbital evolution.

When the orbital plane of the particle is inclined to the equatorial plane of a spinning hole, only one method has been successfully implemented to deduce how radiation reaction influences the evolution of the orbit's "Carter constant" $[4,5]$, which governs the orbital shape and inclination angle. This method, which uses a "post-Newtonian" gravitational radiation reaction force, was described in a previous paper [6], but there only applied to "circular orbits" (orbits of constant Boyer-Lindquist radial coordinate $r$ ) for simplicity. This follow-up paper has a twofold purpose: First, in Sec. II, we will argue that circular orbits remain circular under gravitational radiation reaction. Second, in Sec. III, we will compute the evolution of elliptical orbits under radiation reaction, but only to leading order in $S$, the magnitude of the spin angular momentum of the black hole, and leading order in $M / a$, where $M$ is the black hole's mass and $a$ is the size of the orbit, as defined more precisely below. (Here and throughout, units with $G=c=1$ are used.)

\section{EVOLUTION OF CIRCULAR ORBITS}

Several years ago, Ori [7] put forth the conjecture that circular orbits in the Kerr metric remain circular even under gravitational radiation reaction. Here, we will argue in favor of the conjecture. We will start by reviewing some properties of elliptical and circular orbits in the Kerr metric. Then we will argue that a circular orbit and the reaction force acting on it have a type of reflection symmetry that ensures that the orbit remains circular under radiation reaction, in the limit of the particle's mass being small compared to the hole's mass.

In the absence of gravitational radiation, the geodesic motion of a particle in orbit around a Kerr black hole is well known and discussed, for example, in Sec. 33.6 of Ref. [5]. The location of the particle can be described in BoyerLindquist coordinates $r, \theta, \phi$, and $t$. The orbit can be described by three constants of motion: the energy $E$, the angular momentum along the hole's spin axis $L_{z}$, and the Carter constant $Q$. The particle's rest mass $\mu$ can be counted as another constant of the motion. The energy $E$ is defined as the relativistic energy of the particle minus its rest mass, so that " $E-\mu$ " in the language of Ref. [5] corresponds to " $E$ " here. We will restrict to bound orbits, that is $E<0$ and, as a consequence (see Ref. [4]), $Q \geqslant 0$.

An interesting feature of the Kerr metric in BoyerLindquist coordinates is the existence of nonequatorial, circular, geodesic orbits. These orbits are circular in the sense that the particle maintains a constant Boyer-Lindquist coordinate $r$; however, the plane of the circular orbit is not fixed but rather precesses around the hole's spin axis. Such orbits exist and are stable for values of $E, L_{z}$, and $Q$ that give $R=0, \partial R / \partial r=0$, and $\partial^{2} R / \partial r^{2}<0$, where $R$ [see Eq. (33.33c) of Ref. [5]] is defined by

$$
\begin{aligned}
R= & {\left[(E+\mu)\left(r^{2}+S^{2} / M^{2}\right)-L_{z} S / M\right]^{2}-\left(r^{2}-2 M r+S^{2} / M^{2}\right) } \\
& \times\left[\mu^{2} r^{2}+\left(L_{z}-S \mu / M-S E / M\right)^{2}+Q\right] .
\end{aligned}
$$

For an arbitrary orbit with constants $E, L_{z}$, and $Q$, there might be some other energy $\bar{E} \leqslant E$ ( $\bar{E}$ depends on $L_{z}$ and $Q)$ such that, if the orbit had energy $\bar{E}\left(L_{z}, Q\right)$ rather than $E$, the orbit would be circular and stable. In such a case, as an alternative set of constants to $E, L_{z}$, and $Q$, the constants $\iota, e$, and $a$ can be defined as

$$
\begin{gathered}
\cos \iota \equiv \frac{L_{z}}{\left(Q+L_{z}^{2}\right)^{1 / 2}}, \\
1-e^{2} \equiv \frac{E}{\bar{E}},
\end{gathered}
$$




$$
a \equiv \frac{\bar{E} \bar{r}}{E}
$$

Here $\bar{r}=\bar{r}\left(L_{z}, Q\right)$ is the radius of the circular orbit with constants $\bar{E}, L_{z}$, and $Q$. Note that $a$ should not be confused with the conventional notation for the spin of the black hole, which is $S$ here.

The positive root in Eq. (2a) or in any other square root is always chosen. We choose the angle $\iota$ to lie in the range $0 \leqslant \iota \leqslant \pi$, so that $\iota<\pi / 2$ corresponds to an orbit corotating relative to the spin and $\iota>\pi / 2$ to counterrotating. Also, $e$ is chosen as non-negative.

This set of constants $\iota, e$, and $a$ has the conceptual advantage that in the Newtonian limit of large $a$, the orbit of the particle is an ellipse of eccentricity $e$ and semimajor axis length $a$, on a plane with inclination angle $\iota$ to the hole's equatorial plane. When not in the Newtonian limit, interpreting $\iota, e$, and $a$ as the inclination angle, eccentricity, and semimajor axis length must be done with the caveat that since the orbit is not an ellipse; then, words such as "eccentricity" are subject to a modified interpretation and can be misleading.

Even though the particle's motion is complicated when not in the Newtonian limit, some of the parameters that describe the particle's motion need not be specified. For example, we are not concerned with the value of $\phi$ or $t$, because making a $\phi$ or $t$ translation does not change the physics in the axisymmetric, stationary Kerr metric. Another symmetry is that if the orbital motion is flipped over the hole's equatorial plane, i.e., $\theta(t)$ is replaced by $\pi-\theta(t)$, the motion can be considered the same. All such $\phi$ and $t$ translations and $\theta$ reflections leave the shape of the orbit unchanged.

We can think of the particle as undergoing oscillatory, coupled motion in the $r$ and $\theta$ directions. We define one orbital revolution to be one oscillation cycle as measured by the $\theta$ motion. Given any chosen starting point of an orbital revolution with coordinate $\theta_{0}$, the revolution can be broken into two half revolutions, the first when the particle goes from $\theta_{0}$ to $\pi-\theta_{0}$ half a $\theta$ cycle later, and the second when the particle goes from $\pi-\theta_{0}$ back to $\theta_{0}$ another half $\theta$ cycle later. [Because of the coupling of the $r$ motion with the $\theta$ motion, the $\theta$ motion does not peak at the same extrema every cycle. Therefore, $\left|\pi / 2-\theta_{0}\right|$ has to be chosen small enough that the orbit does indeed go through $\pi-\theta_{0}$ and $\theta_{0}$ in the following cycle. However, this is a very minor restriction for the rest of Sec. II, where in proving that circular orbits stay circular, we only consider circular and almost circular orbits (we do not have to consider generally eccentric orbits since we know that a circular orbit cannot immediately become generally eccentric without first being slightly eccentric). In such case, the peaks of the $\theta$ motion are almost the same every cycle.]

Now we consider the effect of gravitational radiation reaction on an orbit. We assume that the rest mass $\mu$ is small enough for the adiabatic approximation to hold: The time scale of the gravitational radiation reaction is much longer than any other time scale in the problem. Then the particle moves very nearly on a geodesic path characterized by the constants of motion $\iota, e$, and $a$, and only on a very long time scale (which varies like $1 / \mu$ as $\mu \rightarrow 0$, because the radiation reaction acceleration scales like $\mu$ ) is this motion substantially modified by gravitational radiation reaction.

We now consider, for an orbit slowly inspiraling due to radiation reaction, an orbital revolution that satisfies the following condition, to which we give the name reflection symmetry: Consider the point on the orbit that is at the beginning of the orbital revolution. Denote by $r_{0}, \theta_{0}, \dot{r}_{0}, \dot{\theta}_{0}$, and $\dot{\phi}_{0}$ the Boyer-Lindquist spatial coordinates of that point and their time derivatives. (Here and throughout, an overdot represents $d / d t$.) Then there are two other locations later on the path with coordinates

$$
\begin{gathered}
r_{n}=r_{0}+\mu n \tilde{r}+\mathrm{HO}, \\
\theta_{n}-\pi / 2=(-1)^{2 n}\left(\theta_{0}-\pi / 2+\mu n \tilde{\theta}\right)+\mathrm{HO}, \\
\dot{r}_{n}=\dot{r}_{0}+\mu n \dot{\tilde{r}}+\mathrm{HO}, \\
\dot{\theta}_{n}=\dot{\theta}_{0}+\mu n \dot{\tilde{\theta}}+\mathrm{HO}, \\
\dot{\phi}_{n}=\dot{\phi}_{0}+\mu n \dot{\tilde{\phi}}+\mathrm{HO},
\end{gathered}
$$

for $n=\frac{1}{2}$ (a half revolution after $n=0$ ) and $n=1$ (a full orbital revolution after $n=0$ ). The functions with tildes are not functions of $\mu$. The "HO" terms are any terms that go to zero faster than $\mu$ as $\mu \rightarrow 0$ (higher order in $\mu$ than linear).

Because of the initial conditions at the beginning of the first and second half revolutions (at $n=0$ and $n=\frac{1}{2}$, respectively), the shape of the first half revolution (the path connecting the $n=0$ and $n=\frac{1}{2}$ locations) deviates from the shape of the second half revolution (the path connecting the $n=\frac{1}{2}$ and $n=1$ locations) by a path deviation of order $\mu$. Of course, these two paths also differ by a $\phi$ translation, a $t$ translation, and a reflection across the equatorial plane. But as we discussed above, these are unimportant differences because of the symmetries; the shapes of the paths are the same.

Now that we have written Eqs. (3), we temporarily (for the remainder of this paragraph) go back to the case of no radiation reaction; i.e., we set to zero the $\mu$ terms and the HO terms in Eqs. (3). Clearly, a circular orbital revolution satisfies Eqs. (3) for any initial $n=0$ location chosen on the circular orbit. But could there be an eccentric orbit which also satisfies Eqs. (3)? The answer is negative, as we shall now show. A slightly eccentric orbit (one with the value of $e$ small enough that $e^{2}$ terms are negligible) would have the same $\theta(t)$ and $\phi(t)$ motion regardless of the value of $e$, but $r-\bar{r}$ would oscillate with an amplitude proportional to $e$. This can be verified from the Kerr-metric geodesic equations, Eqs. (33.32) of Ref. [5]. In the Newtonian limit, the oscillation of $r-\bar{r}$ would be periodic with the same period that $\theta(t)$ has, but when not in the Newtonian limit the $\theta$ and $r$ oscillations would have different periods. If an orbit were to be reflection symmetric, then $r-\bar{r}$ would have to have the same value when the orbital motion is at $\theta_{0}$ as it does when it is at $\pi-\theta_{0}$ at the next value of $n$. This would require that either $r-\bar{r}$ oscillate at a frequency that is an even integer multiple of the $\theta$ oscillation frequency or $r-\bar{r}$ have zero amplitude (a circular orbit). The former is never the case, as can be verified by numerically [8] examining circular orbits 
in the Kerr metric over the space of possible physically acceptable values of $S, L_{z}$, and $Q$. The fact that $r-\bar{r}$ does not resonate with an even multiple of the $\theta$ frequency implies that a slightly eccentric orbit cannot be reflection symmetric.

Now we shall return to the case of interest: that with gravitational radiation reaction. What precisely do we refer to when we discuss circular orbital revolutions, when the orbital revolution is not actually circular but is slowly inspiraling? A good, but not unique, definition is one that agrees with the result in the case of no radiation reaction: We define that a circular orbital revolution is one that satisfies Eqs. (3), while an eccentric orbital revolution is one that does not (at least for slight eccentricity, as mentioned above, we are not considering generally eccentric orbits). An orbital revolution with weak radiation reaction is defined as circular if and only if it is reflection symmetric.

We now consider starting with an initial orbital revolution that is circular or, equivalently, that is reflection symmetric, i.e., that satisfies conditions (3). For small $\mu$, ignoring the HO (higher than $\mu$ ) corrections, we would expect that the third half revolution (the first half of the next orbital revolution) would have a shape that deviates from that of the second half revolution by the same amount as the shape of the second half revolution deviates from that of the first. We expect this, because from conditions (3) above, the initial conditions of the third half revolution differ from those of the second by the same amount (to linear order in $\mu$ ) as those of the second differ from those of the first, and the acceleration on the particle should similarly be equally (also to linear order in $\mu$ ) different between corresponding locations on the second and third half revolutions as between corresponding locations on the first and second. The orbit remains circular for the additional half revolution. If there is any eccentricity added, it is in the HO terms, but in the $\mu \rightarrow 0$ limit, this is ignorable compared to the shrinking of the orbit, which varies like $\mu$ (the terms involving tildes).

We can repeat the above argument to get the shape of the fourth half revolution, as well as the fifth, sixth, etc. In fact, the argument can be repeated to any chosen number $n_{\max }$ of orbital revolutions, as long as that chosen number does not go to infinity as $\mu \rightarrow 0$; for if it did, then we would not be guaranteed that after an infinite number $n_{\max }$ of orbits, the $\mathrm{HO}$ corrections of the above paragraph would be ignorable. For example, we could choose $n_{\max }$ to be 100 , but we could not choose it to be $100 \mathrm{M} / \mu$. The orbit remains reflection symmetric (or, equivalently, it remains circular) for $n$ up to $n_{\max }$, where $n$ increments by $\frac{1}{2}$. In other words, there is a location, with coordinates $r_{n}, \theta_{n} \dot{r}_{n}, \dot{\theta}_{n}$, and $\dot{\phi}_{n}$, satisfying Eqs. (3) for any $n$ up to $n_{\max }$.

The constants of motion $E, L_{z}$, and $Q$ (or, equivalently, $\iota, e$, and $a$ ) evolve in such a way that in going from $n=0$ to $n=n_{\text {max }}$ a circular orbit remains circular. By assigning new values of $r_{0}, \theta_{0}, \dot{r}_{0}, \dot{\theta}_{0}$, and $\dot{\phi}_{0}$ as the old $r_{n_{\max }}, \theta_{n_{\max }}$, $\dot{r}_{n_{\max }}, \dot{\theta}_{n_{\max }}$, and $\dot{\phi}_{n_{\text {max }}}$, the argument can be repeated, over and over again. The rates of loss of $E, L_{z}$, and $Q$ will then continue at such a rate so as to maintain circularity.

A more intuitive picture of why a circular orbit remains circular was provided by Ori [7], who first pointed out that the incommensurability of the $r$ and $\theta$ periods is the key reason why the argument can be made without knowing the nature of the reaction force: Even if the radiation reaction were to take the bizarre form of somebody with a hammer hitting the particle every time the particle is at some value of $\theta$, there would have to be another person across the equatorial plane at $\pi-\theta$ with a hammer hitting the particle in a corresponding way, as dictated by the orbital symmetries. Since the $r-\bar{r}$ frequency is not an even multiple of the $\theta$ frequency, the hammer hits cannot constructively interfere with each other and produce an eccentricity.

If an orbit is circular, then just knowing the rates of change of $E$ and $L_{z}$ (for example, by knowing the energy and angular momentum carried off in the gravitational waves) is enough to determine the full orbital evolution since the evolution of $Q$ is constrained such that the conditions listed immediately before Eq. (1) are satisfied, for as long as the orbit itself is stable.

\section{LEADING ORDER EFFECT OF SPIN ON ECCENTRIC ORBITS}

We now wish to consider general, not just circular, orbits around a black hole. But in doing so, we restrict ourselves to only considering the leading order effect of spin. We will use the formalism of a radiation reaction force described in a previous paper [6] and merely state how the method as described in that paper generalizes to orbits with eccentricity.

When one is only interested in leading order in $S$ and in $M / r$ (or, equivalently, $M / a$, in terms of orbit parameters), the effect of the hole's Kerr metric on the particle's motion can be substituted with a spin-orbit interaction in threedimensional flat space. Let spherical polar coordinates $r$, $\theta$, and $\phi$, centered on the black hole, be used to describe the location of the particle (these coordinates describe the relative separation of the two bodies), with the hole's spin along the polar axis. The Lagrangian [Ref. [9], Eq. (4)] for the motion of the particle is given, to linear order in $S$ but otherwise in solely Newtonian theory, by

$$
\mathscr{C}=\frac{\mu}{2}\left[\dot{r}^{2}+r^{2} \dot{\theta}^{2}+r^{2} \sin ^{2}(\theta) \dot{\phi}^{2}\right]+\frac{\mu M}{r}-\frac{2 \mu S \sin ^{2} \theta}{r} \dot{\phi} .
$$

To leading order in $S$ and in $M / r$, the motion resulting from this Lagrangian is the same as in the Kerr metric. The use of flat space coordinates, which ignores $M / r$ corrections, is adequate to leading order. Using the same coordinate variable names $r, \theta$, and $\phi$ for these coordinates as for the Kerr metric's Boyer-Lindquist coordinates does not cause conflict and should not cause confusion. Alternatively, we can use Cartesian coordinates, $x_{1}=r \sin \theta \cos \phi, x_{2}=r \sin \theta \sin \phi$, and $x_{3}=r \cos \theta$.

The Lagrangian (4) admits three constants of motion, called $E, L_{z}$, and $Q$ because they are the same constants as we have in the Kerr metric, to leading order in $S$ and in $M / r$. The values of these constants are

$$
\begin{gathered}
E=\frac{\mu}{2}\left[\dot{r}^{2}+r^{2} \dot{\theta}^{2}+r^{2} \sin ^{2}(\theta) \dot{\phi}^{2}\right]-\frac{\mu M}{r}, \\
L_{z}=\mu r^{2} \sin ^{2}(\theta) \dot{\phi}-\frac{2 \mu S \sin ^{2} \theta}{r},
\end{gathered}
$$




$$
Q+L_{z}^{2}=\mu^{2} r^{4}\left[\dot{\theta}^{2}+\sin ^{2}(\theta) \dot{\phi}^{2}\right]-4 \mu^{2} S r \sin ^{2}(\theta) \dot{\phi} .
$$

The combination $Q+L_{z}^{2}$ is a more natural constant to work with than $Q$. If $S$ were equal to zero, then $Q+L_{z}^{2}$ would be the square of the total angular momentum.

The constants of motion $\iota, e$, and $a$, when considered only to leading order in $S$ and in $M / r$, are related to $E$, $L_{z}$, and $Q$ by

$$
\begin{gathered}
\cos \iota=\frac{L_{z}}{\left(Q+L_{z}^{2}\right)^{1 / 2}}, \\
1-e^{2}=-2 \frac{E\left(Q+L_{z}^{2}\right)}{\mu^{3} M^{2}}\left(1+4 \frac{S M \mu^{3} L_{z}}{\left(Q+L_{z}^{2}\right)^{2}}\right), \\
a=-\frac{M \mu}{2 E}\left(1+2 \frac{S M \mu^{3} L_{z}}{\left(Q+L_{z}^{2}\right)^{2}}\right) .
\end{gathered}
$$

It is easy to verify these, by checking that the $\bar{E}$ and $\bar{r}$ that would make Eqs. (2) give Eqs. (6) satisfy (at leading order in $S$ and in $M / a$ ) the stable circular orbit constraints listed immediately before Eq. (1). Note that Eqs. (6) are valid for arbitrary eccentricity $e$; they do not require $e \ll 1$.

It is possible to express the instantaneous time derivative of each constant of motion, $d E / d t, d L_{z} / d t$, or $d\left(Q+L_{z}^{2}\right) / d t$, as a function of $r, x_{3}, \dot{r}, \dot{x}_{3}$, and the constants of motion; there is no occurrence of $\phi$ (because of the axisymmetry) or $\dot{\phi}$ (as this is determined with $L_{z}, r$, and $\theta$ known) in any of the expressions. If $S$ were zero, then there could be no $x_{3}$ dependence, rather only $r$ dependence, since there is no physically preferred direction when spin is absent. Thus, an $x_{3}$ or $\dot{x}_{3}$ can only show up in a term that includes a factor of $S$. Because of this, to compute the time derivative of each constant of motion to Newtonian order plus the spin correction, $x_{3}(t)$ only needs to be known to Newtonian order, because the spin correction to $x_{3}$ would be an $S^{2}$ term in the derivative of the constant of motion. On the other hand, the radial motion $r(t)$ of the particle has to be known to Newtonian order plus the spin correction. The $\phi(t)$ motion does not have to be known at all for computing the evolution of the constants of motion.

Let us, then, compute $r$ and $x_{3}$ to the necessary orders. One of the Euler-Lagrange equations yields

$$
\ddot{r}=-\frac{M}{r^{2}}+\frac{Q+L_{z}^{2}}{\mu^{2} r^{3}}+6 \frac{S L_{z}}{\mu r^{4}} .
$$

The solution of this, in terms of a parameter $\psi$, is

$$
r=\frac{\left(Q+L_{z}^{2}\right) /\left(\mu^{2} M\right)}{1+e \cos \psi}\left(1+\frac{S L_{z} \mu^{3} M}{\left(Q+L_{z}^{2}\right)^{2}}(6+2 e \cos \psi)\right),
$$

$$
\frac{d t}{d \psi}=\frac{\left(Q+L_{z}^{2}\right)^{3 / 2} /\left(\mu^{3} M^{2}\right)}{(1+e \cos \psi)^{2}}\left(1+6 \frac{S L_{z} \mu^{3} M}{\left(Q+L_{z}^{2}\right)^{2}}\right) .
$$

In the Newtonian limit of $S=0$, these are the equations for a Keplerian ellipse, with the true anomaly $\psi$ being the angle on the orbital plane of the particle relative to periastron as seen from the hole.

To Newtonian order, $x_{3}=r \cos \theta$ can be expressed as

$$
x_{3}=r \sin \iota \sin \left(\psi+\psi_{0}\right)
$$

Here, $\psi_{0}$ is some constant that describes the orientation of the ellipse on the orbital plane. As seen from the hole, $\psi_{0}$ is the angle between the direction of the periastron and the intersection of the equatorial and orbital planes.

The orbital period, from periastron to periastron, is

$$
T=\int_{0}^{2 \pi} d \psi \frac{d t}{d \psi}=2 \pi M\left(\frac{\mu}{-2 E}\right)^{3 / 2}
$$

It happens that $T$, when written in this form, does not have an explicit $S$ dependence.

This motion we have just described is that in the absence of gravitational radiation reaction; now we will compute the effect of the radiation reaction acceleration. We can take the equations for the rates of change of $E, L_{z}$, and $Q$ due to radiation reaction for a particle going around a more massive spinning body from Eqs. (10), (13), and (14) of Ref. [6]. These equations give us formulas for $\dot{E}, \dot{L}_{z}$, and $d\left(Q+L_{z}^{2}\right) / d t$ as functions of the displacement of the particle relative to the hole in Cartesian coordinates, $x_{k}$, and the relative velocity, $\dot{x}_{k}$. There will also be higher order time derivatives of $x_{k}$ (such as $\ddot{x}_{k}, \dddot{x}_{k}$, etc.), but these derivatives can be eliminated from the expressions for $\dot{E}, \dot{L}_{z}$, and $\dot{Q}+L_{z}^{2}$ with the aid of the Euler-Lagrange equations [derived from (4) when expressed in Cartesian coordinates-note that repeated indices are summed over 1,2,3]:

$\ddot{x}_{k}=-\frac{M}{r^{3}} x_{k}+S\left(-\frac{4}{r^{3}} \epsilon_{3 k j} \dot{x}_{j}+6 \frac{\dot{r}}{r^{4}} \epsilon_{3 k j} x_{j}+6 \frac{L_{z}}{\mu r^{5}} x_{k}\right)$.

The time evolution of each constant of motion can thereby be expressed in terms of $r, \dot{r}, x_{3}, \dot{x}_{3}$, and the constants of motion. The trajectory (8)-(11) can be inserted into these expressions, and then time averaged using

$$
\langle\dot{E}\rangle=\frac{1}{T} \int_{0}^{2 \pi} d \psi \frac{d t}{d \psi} \dot{E}
$$

and similarly for $L_{z}$ and $Q+L_{z}^{2}$. The result is [8]

$$
\langle\dot{E}\rangle=-\frac{32}{5} \frac{\mu^{2}}{M^{2}}\left(\frac{M}{a}\right)^{5}\left(\frac{1}{1-e^{2}}\right)^{7 / 2}\left[\left(1+\frac{73}{24} e^{2}+\frac{37}{96} e^{4}\right)-\frac{S}{M^{2}}\left(\frac{M}{a\left(1-e^{2}\right)}\right)^{3 / 2} \cos \iota\left(\frac{73}{12}+\frac{1211}{24} e^{2}+\frac{3143}{96} e^{4}+\frac{65}{64} e^{6}\right)\right],
$$




$$
\begin{aligned}
\left\langle\dot{L}_{z}\right\rangle= & -\frac{32}{5} \frac{\mu^{2}}{M}\left(\frac{M}{a}\right)^{7 / 2}\left(\frac{1}{1-e^{2}}\right)^{2}\left[\cos \iota\left(1+\frac{7}{8} e^{2}\right)+\frac{S}{M^{2}}\left(\frac{M}{a\left(1-e^{2}\right)}\right)^{3 / 2}\left(\left[\frac{61}{24}+\frac{63}{8} e^{2}+\frac{95}{64} e^{4}\right]-\cos ^{2} \iota\left[\frac{61}{8}+\frac{109}{4} e^{2}+\frac{293}{64} e^{4}\right]\right.\right. \\
& \left.\left.-\cos \left(2 \psi_{0}\right) \sin ^{2} \iota\left[\frac{5}{4} e^{2}+\frac{13}{16} e^{4}\right]\right)\right] \\
\left\langle\dot{Q}+L_{z}^{2}\right\rangle= & -\frac{64}{5} \mu^{3}\left(\frac{M}{a}\right)^{3}\left(\frac{1}{1-e^{2}}\right)^{3 / 2}\left[\left(1+\frac{7}{8} e^{2}\right)-\frac{S}{M^{2}}\left(\frac{M}{a\left(1-e^{2}\right)}\right)^{3 / 2} \cos \iota\left(\frac{97}{12}+22 e^{2}+\frac{99}{32} e^{4}\right)\right]
\end{aligned}
$$

The evolution of these constants can be converted, using Eqs. (6), to the other set of constants:

$$
\begin{array}{r}
\langle i\rangle=\frac{\mu S}{M^{4}}\left(\frac{M}{a}\right)^{11 / 2}\left(\frac{1}{1-e^{2}}\right)^{4} \sin \iota\left[\frac{244}{15}+\frac{252}{5} e^{2}+\frac{19}{2} e^{4}-\cos \left(2 \psi_{0}\right)\left(8 e^{2}+\frac{26}{5} e^{4}\right)\right], \\
\langle\dot{a}\rangle=-\frac{64}{5} \frac{\mu}{M}\left(\frac{M}{a}\right)^{3}\left(\frac{1}{1-e^{2}}\right)^{7 / 2}\left[\left(1+\frac{73}{24} e^{2}+\frac{37}{96} e^{4}\right)-\frac{S}{M^{2}}\left(\frac{M}{a\left(1-e^{2}\right)}\right)^{3 / 2} \cos \iota\left(\frac{133}{12}+\frac{337}{6} e^{2}+\frac{2965}{96} e^{4}+\frac{65}{64} e^{6}\right)\right], \\
\langle\dot{e}\rangle=-\frac{\mu}{M^{2}}\left(\frac{M}{a}\right)^{4}\left(\frac{1}{1-e^{2}}\right)^{5 / 2} e\left[\frac{304+121 e^{2}}{15}-\frac{S}{M^{2}}\left(\frac{M}{a\left(1-e^{2}\right)}\right)^{3 / 2} \cos \iota\left(\frac{1364}{5}+\frac{5032}{15} e^{2}+\frac{263}{10} e^{4}\right)\right] .
\end{array}
$$

Equations (14) agree (after trivial conversions of notation) with previous results: Eqs. (15) of Ref. [6] and (the first line of) Eq. (3.14) of Ref. [10], each of which is a special case of Eqs. (14).

In most cases, the terms with the $\cos \left(2 \psi_{0}\right)$ can be dropped because they average to zero; to see when this can be done, consider the following: The Newtonian approximation to the motion is that the particle travels in an ellipse. The first correction to this motion is, as Einstein computed for Mercury, that the periastron position of the ellipse shifts on a time scale of

$$
T_{\mathrm{prec}} \sim M(M / a)^{-5 / 2}\left(1-e^{2}\right)
$$

The radiation reaction time scale for terms that involve $\psi_{0}$, as computed by evaluating $(\sin \iota) /\langle i\rangle_{\psi_{0}}$ terms, is

$$
T_{\mathrm{rad}} \sim M\left(\frac{M}{a}\right)^{-11 / 2}\left(\frac{S}{M^{2}}\right)^{-1}\left(\frac{\mu}{M}\right)^{-1} \frac{\left(1-e^{2}\right)^{4}}{e^{2}} .
$$

(There are also factors of order unity that involve $e$ which were ignored. If $T_{\text {rad }}$ were computed differently, for example by evaluating $L_{z} /\left\langle\dot{L}_{z}\right\rangle_{\psi_{0}}$ terms, it would contain factors of $\iota$ as well.)

In the Newtonian limit, $\psi_{0}$ is fixed, but with the periastron precession, $\psi_{0}$ changes slightly after each orbit, by a postNewtonian correction that was ignorable until now: When $T_{\text {rad }} \gg T_{\text {prec }}$, the $\cos \left(2 \psi_{0}\right)$ in Eqs. (14b) and (15a) averages to zero, and the terms with that factor can be dropped. For extremely eccentric orbits, $T_{\text {rad }}$ might not be much greater than $T_{\text {prec }}$, and so the $\psi_{0}$ terms must be kept. In all other respects, the periastron precession can be ignored because it just gives terms higher order in $M / a$ (terms we have ne- glected). The only effect of the precession, to which our analysis is sensitive, is the averaging away of $\psi_{0}$ in the case that $T_{\text {rad }} \gg T_{\text {prec }}$.

From Eq. (15a), it is clear that the angle $\iota$ changes such as to become antialigned with the spin. In Ref. [6], this conclusion was reached for circular orbits; finite eccentricity does not change, but only enhances, this result. However, the statement that "the inclination angle antialigns with the spin" is subject to the warning that we mentioned above when introducing $\iota$ : With the orbit not confined to a fixed plane, the angle $\iota$ is not the only way we could define "inclination angle" [6].

Equation (15c) has two important consequences: First, to leading order, orbits tend to circularize, as is a well-known fact. Second, if an orbit is circular, then $e=0$ and $\langle\dot{e}\rangle=0$, and so the orbit remains circular. This is expected, since this is the leading order limit of the general result in Sec. II.

The above analysis is just one step in a general program for understanding the effects of radiation reaction on orbiting, spinning bodies. Future steps in this program include generalizing the analysis to an arbitrary mass ratio $\mu / M$ and to the case of both masses having spin, extending the analysis to higher order in $M / r$ and in $S$, and achieving a similar calculation of the orbital evolution in the fully relativistic Kerr metric.

\section{ACKNOWLEDGMENTS}

The author is grateful to Daniel Kennefick, Alan Wiseman, and Kip Thorne for their advice. This work was supported by NSF Grant No. AST-9417371, and by NASA Grant No. NAGW-4268. 
[1] A. Abramovici, W. E. Althouse, R. W. P. Drever, Y. Gürsel, S. Kawamura, F. J. Raab, D. Shoemaker, L. Sievers, R. E. Spero, K. S. Thorne, R. E. Vogt, R. Weiss, S. E. Whitcomb, and M. E. Zucker, Science 256, 325 (1992).

[2] C. Bradaschia, E. Calloni, M. Cobal, R. Del Fasbro, A. Di Virgilio, A. Giazotto, L. E. Holloway, H. Kautzky, B. Michelozzi, V. Montelatici, D. Pascuello, and W. Velloso, in Gravitation: 1990, Proceedings of the Banff Summer Institute, Banff, Alberta, 1990, edited by R. Mann and P. Wesson (World Scientific, Singapore, 1991).

[3] K. Danzmann, A. Rüdiger, R. Schilling, W. Winkler, J. Hough, G. P. Newton, D. Robertson, N. A. Robertson, H. Ward, P. Bender, J. Faller, D. Hils, R. Stebbins, C. D. Edwards, W. Folkner, M. Vincent, A. Bernard, B. Bertotti, A. Brillet, C. N. Man, M. Cruise, P. Gray, M. Sandford, R. W. P. Drever, V. Kose, M. Kühne, B. F. Schutz, R. Weiss, and H. Welling, LISA: Proposal for a Laser-Interferometric Gravitational Wave Detector in Space (Max-Planck-Institut für Quantenop- tik, Garching bei München, Germany, 1993).

[4] B. Carter, Phys. Rev. 174, 1559 (1968).

[5] C. W. Misner, K. S. Thorne, and J. A. Wheeler, Gravitation (Freeman, San Francisco, 1973).

[6] F. D. Ryan, Phys. Rev. D 52, R3159 (1995).

[7] Ori's original argument is unpublished, but a revised argument will be presented by D. Kennefick and A. Ori [Phys. Rev. D (to be published)]. The author has also recently become aware of another argument that circular orbits remain circular by Y. Mino (in preparation).

[8] Algebraic manipulations were performed with the aid of MATHEMATICA [S. Wolfram, Mathematica: A System for Doing Mathematics by Computer (Addison-Wesley, Redwood City, California, 1988)].

[9] L. E. Kidder, C. M. Will, and A. G. Wiseman, Phys. Rev. D 47, R4183 (1993).

[10] M. Shibata, Phys. Rev. D 50, 6297 (1994). 